\title{
A stochastic pitchfork bifurcation in a reaction-diffusion equation
}

\author{
By Tomás Caraballo, José A. Langa and James C. Robinson† \\ Departamento de Ecuaciones Diferenciales y Análisis Numérico, \\ Universidad de Sevilla, Apdo. de Correos 1160, 41080-Sevilla, Spain
}

Received 10 July 2000; revised 12 January 2001; accepted 15 March 2001

We study in some detail the structure of the random attractor for the Chafee-Infante reaction-diffusion equation perturbed by a multiplicative white noise,

$$
\mathrm{d} u=\left(\Delta u+\beta u-u^{3}\right) \mathrm{d} t+\sigma u \circ \mathrm{d} W_{t}, \quad x \in D \subset \mathbb{R}^{m} .
$$

First we prove, for $m \leqslant 5$, a lower bound on the dimension of the random attractor, which is of the same order in $\beta$ as the upper bound we derived in an earlier paper, and is the same as that obtained in the deterministic case. Then we show, for $m=1$, that as $\beta$ passes through $\lambda_{1}$ (the first eigenvalue of the negative Laplacian) from below, the system undergoes a stochastic bifurcation of pitchfork type. We believe that this is the first example of such a stochastic bifurcation in an infinite-dimensional setting. Central to our approach is the existence of a random unstable manifold.

Keywords: random attractors; stochastic bifurcation; Hausdorff dimension

\section{Introduction}

Attractors for infinite-dimensional dynamical systems have proven to be a very useful tool in the study of the asymptotic behaviour of many partial differential equations (see, for example, Hale 1988; Ladyzhenskaya 1991; Temam 1988). Much attention has been focused on the dimension of these attractors, since in many cases one can prove that this is finite, and deduce that the long-time behaviour of the system depends on only a finite number of degrees of freedom.

The definition of an attractor has been generalized to the stochastic case by Crauel \& Flandoli (1994), Crauel et al. (1997) and Schmalfuß (1992), and has once again been fruitful in furthering our understanding of the associated random and stochastic differential equations, in particular in finite dimensions (Arnold 1998).

Roughly speaking, a random attractor is a family of compact random sets, that is invariant for the stochastic flow and attract all solutions 'from $t=-\infty$ '. Once again, it is possible to show that a certain number of these attractors are finite dimensional, although the number of examples is much more limited than in the deterministic case. Debussche (1998) has adapted the most powerful deterministic technique to treat the stochastic case, and in a previous paper (Caraballo et al. 2000) we used his method to obtain an upper bound on the Hausdorff dimension of the random attractor for

$\dagger$ Permanent address: Mathematics Institute, University of Warwick, Coventry CV4 7AL, UK. 
the Chafee-Infante reaction-diffusion equation perturbed by a multiplicative noise in the sense of Stratonovich,

$$
\mathrm{d} u=\left(\Delta u+\beta u-u^{3}\right) \mathrm{d} t+\sigma u \circ \mathrm{d} W_{t}, \quad x \in D \subset \mathbb{R}^{m} .
$$

Different techniques are required, for both stochastic and deterministic systems, to obtain lower bounds on the dimension of these attractors. Such bounds are generally based on finding some invariant manifolds, which must be subsets of the attractor (Babin \& Vishik 1983).

Here we adopt this approach, using some ideas due to Da Prato \& Debussche (1996), to show (in $\S 4$ ) that there is an $n$-dimensional unstable manifold near the zero solution when $\lambda_{n}<\beta<\lambda_{n+1}$ (the $\lambda_{j}$ are the eigenvalues of the negative Laplacian, see $\S 3$ ). This gives a lower bound on the attractor dimension, which is of the same order as our previous upper bound. Remarkably, these bounds do not depend on the level of the noise $(\sigma$ in $(1.1)$ ), and are of the same order as the bounds in the deterministic case.

In the last decade there has been some research in stochastic bifurcation theory, although it still seems to be unclear how this theory can be set up in general (Arnold 1998). However, to our knowledge, there are as yet no studies of bifurcations in infinite-dimensional stochastic differential equations. We adopt here the dynamical concept of a stochastic bifurcation, which is understood as a qualitative change in the invariant sets (or invariant measures) of the system (see $\S 5$ ).

For the one-dimensional case $m=1$, we use the manifold structure, which gave us the lower bound on the dimension, to investigate in more detail what happens to the random attractor as $\beta$ passes through $\lambda_{1}$ from below. For $\beta<\lambda_{1}$, we showed previously that the attractor is just the point $\{0\}$, and our lower bounds show that for $\beta>\lambda_{1}$ the Hausdorff dimension of the attractor is at least 1. We show that for $\lambda_{1}<\beta<\lambda_{2}$ the unstable manifold is, in fact, tangent to the space spanned by the first eigenfunction of the Laplacian, and thus actually has one branch in the cone of positive solutions and the other one in the cone of negative solutions (see $\S 5$ ). It is only a short step from here to the required pitchfork bifurcation, using the theory of monotonic random dynamical systems developed by Arnold \& Chueshov (1998).

We end with some conclusions and open problems.

\section{Formulation of the problem and deterministic results}

Let $D \subset \mathbb{R}^{m}, m \leqslant 5$, be an open bounded set with regular boundary. We consider the following Chafee-Infante reaction-diffusion equation in $D$ perturbed by a linear multiplicative white noise,

$$
\mathrm{d} u=\left(\Delta u+\beta u-u^{3}\right) \mathrm{d} t+\sigma u \circ \mathrm{d} W_{t},
$$

with $u(x, t)=0$ for $x \in \partial D$, and where $W .(\omega): \Omega \rightarrow C^{0}(\mathbb{R}, \mathbb{R})$ is a one-dimensional Wiener process on the probability space $(\Omega, \mathcal{F}, \mathbb{P})$.

We rewrite (2.1) as the following differential equation on $L^{2}(D)$,

$$
\mathrm{d} u=\left(-A u+\beta u-u^{3}\right) \mathrm{d} t+\sigma u \circ \mathrm{d} W_{t},
$$

where $A=-\Delta$ on $D$ with the appropriate (Dirichlet) boundary conditions. The operator $A$ is positive, linear, self-adjoint and has compact inverse. Thus there exists 
a sequence $\left\{\lambda_{j}\right\}$ of positive eigenvalues, whose associated eigenfunctions $w_{j}$ (with $A w_{j}=\lambda_{j} w_{j}$ ) form an orthonormal basis for $H$ (see, for example, Renardy \& Rogers 1992). We order these so that $\lambda_{n+1} \geqslant \lambda_{n}$.

\section{(a) The deterministic case $(\sigma=0)$}

When $\sigma=0$, existence and uniqueness results are proved for the equation in Marion (1987), Temam (1988) and Robinson (2001). There exists a unique weak solution

$$
u\left(t ; u_{0}\right) \in L^{2}\left(0, T ; H_{0}^{1}(D)\right) \cap L^{4}((0, T) \times D) \cap C\left([0, T] ; L^{2}(D)\right),
$$

so that, in particular, we can use the solutions to define a semigroup $S(t)$ on $L^{2}(D)$, via

$$
S(t) u_{0}=u\left(t ; u_{0}\right)
$$

$S(t)$ satisfies the usual semigroup properties,

$$
S(0)=\mathrm{id}, \quad S(t) S(s)=S(t+s) \quad \text { and } \quad S(t) u_{0} \text { continuous in } t \text { and } u_{0} .
$$

It is shown in all three of the above references that the equation also enjoys the existence of a global attractor $\mathcal{A}$, that is, a compact invariant set that attracts the orbits of all bounded sets, i.e.

$$
S(t) \mathcal{A}=\mathcal{A} \quad \text { for all } t \in \mathbb{R}
$$

and

$$
\operatorname{dist}(S(t) B, \mathcal{A}) \rightarrow 0 \text { as } t \rightarrow \infty
$$

where $B$ is any bounded subset of $L^{2}(D)$ and $\operatorname{dist}(A, B)$ is the Hausdorff semidistance between $A$ and $B$,

$$
\operatorname{dist}(A, B)=\sup _{a \in A} \inf _{b \in B}|a-b| .
$$

Estimates of the Hausdorff dimension of this attractor in terms of $\beta$ can also be obtained, namely

$$
d_{\mathrm{H}}(\mathcal{A}) \leqslant C \beta^{m / 2}
$$

(Recall that $D \subset \mathbb{R}^{m}$.) By showing the existence of an unstable manifold near the origin, Babin \& Vishik (1983) show that a similar lower bound holds, so that, in fact, there exists a constant $c$ such that

$$
c \beta^{m / 2} \leqslant d_{\mathrm{H}}(\mathcal{A}) \leqslant C \beta^{m / 2}
$$

(see also Temam 1988). We write this more compactly as

$$
d_{\mathrm{H}}(\mathcal{A})=O\left(\beta^{m / 2}\right) .
$$

We will obtain, below, the same behaviour in the stochastic case.

In the particular case $m=1$, the structure of this attractor is extremely well understood: the attractor consists of a collection of stationary points, which bifurcate from the origin as $\beta$ passes through each eigenvalue $\lambda_{j}$, and the unstable manifolds joining them (Hale 1988; Henry 1984). In particular, as $\beta$ passes through $\lambda_{1}$, the attractor changes from a single stable fixed point at $u \equiv 0$ to a set, homeomorphic 
to an interval, which consists of the one-dimensional unstable manifold of the origin. This has two distinct components, one of which lies in the cone of positive solutions and on which all solutions approach a new positive fixed point, and one which lies in the cone of negative solutions and on which all solutions approach a new negative fixed point. It is this pitchfork bifurcation that we will seek to mirror in the stochastic case.

\section{Random attractors}

We now briefly discuss the definition of a random dynamical system and a random attractor, unsurprisingly using our equation (2.2) as an illustrative example.

\section{(a) Random dynamical systems}

Let $(\Omega, \mathcal{F}, \mathbb{P})$ be a probability space and $\left\{\theta_{t}: \Omega \rightarrow \Omega, t \in \mathbb{R}\right\}$ a family of measure-preserving transformations such that $(t, \omega) \mapsto \theta_{t} \omega$ is measurable, $\theta_{0}=\mathrm{id}$, and $\theta_{t+s}=\theta_{t} \theta_{s}$ for all $s, t \in \mathbb{R}$. The flow $\theta_{t}$, together with the corresponding probability space

$$
\left(\Omega, \mathcal{F}, \mathbb{P},\left(\theta_{t}\right)_{t \in \mathbb{R}}\right),
$$

is called a (measurable) dynamical system.

In our case, we take $(\Omega, \mathcal{F}, \mathbb{P})$ to be the probability space that generates the onedimensional Wiener process $\mathrm{d} W_{t}$. The shift $\theta_{t}$ acts on $\Omega$, so that

$$
W_{t}\left(\theta_{s} \omega\right)=W_{t+s}(\omega)-W_{s}(\omega),
$$

the additional subtracted term ensuring that $W .\left(\theta_{s} \omega\right)$ is still a Brownian motion. For this example, it also follows that the shift $\theta_{t}$ is ergodic (Arnold 1998).

A continuous random dynamical system (RDS) on a Polish space $(X, d)$ with Borel $\sigma$-algebra $\mathcal{B}$ over $\theta$ on $(\Omega, \mathcal{F}, \mathbb{P})$ is a measurable map

$$
\begin{aligned}
\varphi: \mathbb{R}^{+} \times \Omega \times X & \rightarrow X, \\
(t, \omega, x) & \mapsto \varphi(t, \omega) x,
\end{aligned}
$$

such that, $\mathbb{P}$-almost surely ( $\mathbb{P}$-a.s. $)$,

(i) $\varphi(0, \omega)=$ id on $X$,

(ii) $\varphi(t+s, \omega)=\varphi\left(t, \theta_{s} \omega\right) \circ \varphi(s, \omega)$ for all $t, s \in \mathbb{R}^{+}$(cocycle property),

(iii) $\varphi(t, \omega): X \rightarrow X$ is continuous.

When $\sigma \neq 0$, it is known (Pardoux 1975) that for each $u_{0} \in L^{2}(D)$ and $T>0$ there exists a unique solution $u\left(t ; x_{0}\right)$ of $(2.2)$, with

$$
u\left(t ; x_{0}\right) \in L^{2}\left(\Omega \times(0, T) ; H_{0}^{1}(D)\right) \cap L^{4}(\Omega \times(0, T) \times D) \cap L^{2}\left(\Omega ; C\left(0, T ; L^{2}(D)\right)\right) .
$$

In particular, it follows that the solutions of (2.2) can be used to generate a random dynamical system if we define

$$
\varphi(t, \omega) u_{0}=u\left(t ; \omega, u_{0}\right),
$$

where $u\left(t ; \omega, u_{0}\right)$ is the solution to $(2.2)$ with noise $\omega$ and initial condition $u(0)=u_{0}$. 


\section{(b) Random attractors}

A random set $\mathcal{A}(\omega)$ is said to be a random attractor for the $\operatorname{RDS} \varphi$ if the following hold.

(i) $\mathcal{A}(\omega)$ is a random compact set, that is, $\mathbb{P}$-a.s., $\mathcal{A}(\omega)$ is compact and for all $x \in X$, and the map $\omega \mapsto \operatorname{dist}(x, \mathcal{A}(\omega))$ is measurable with respect to $\mathcal{F}$.

(ii) $\mathbb{P}$-a.s., $\varphi(t, \omega) \mathcal{A}(\omega)=\mathcal{A}\left(\theta_{t} \omega\right)$ for all $t \geqslant 0$ (invariance).

(iii) For all $B \subset X$ bounded (and non-random), $\mathbb{P}$-a.s.,

$$
\lim _{t \rightarrow \infty} \operatorname{dist}\left(\varphi\left(t, \theta_{-t} \omega\right) B, \mathcal{A}(\omega)\right)=0,
$$

where $\operatorname{dist}(\cdot, \cdot)$ denotes the Hausdorff semi-distance in $X$ (cf. (2.6)).

Since $\varphi\left(t, \theta_{-t} \omega\right) u_{0}$ can be interpreted as the position at $t=0$ of the trajectory that was at $u_{0}$ at time $-t$, this pull-back convergence property is essentially attraction 'from $t=-\infty$ '.

In Caraballo et al. (2000), we proved the existence of a random attractor for our equation, using a theorem due to Crauel \& Flandoli (1994). We also showed that if $\beta<\lambda_{1}$, then $\mathcal{A}(\omega)=\{0\}$ and, more generally (using a result of Debussche (1998)), that if

$$
\beta<\frac{1}{d} \sum_{j=1}^{d} \lambda_{j},
$$

then $d_{\mathrm{H}}(\mathcal{A}(\omega))<d$. Since one can bound $\sum_{j=1}^{d} \lambda_{j} \leqslant C d^{(m+2) / m}$, this implies the upper bound

$$
d_{\mathrm{H}}(A(\omega)) \leqslant c_{1} \beta^{m / 2} .
$$

Note that this is of the same order as the deterministic bound in (2.7), and of course is extremely suggestive of the fact that the attractor becomes more complicated as $\beta$ increases through $\lambda_{1}$.

\section{A lower bound on the attractor dimension}

We now turn to proving the promised lower bound on the dimension of the random attractor $\mathcal{A}(\omega)$. We make use of an idea from the deterministic theory, which is to show that the attractor must contain an unstable manifold of a certain dimension. However, this approach usually requires various differentiability properties of the flow, and such technicalities are by no means straightforward in the stochastic case. Furthermore, there is currently no well-developed theory of unstable manifolds for general stochastic partial differential equations (PDEs).

So we proceed in a manner that we believe is slightly unusual, and could prove useful not only for other stochastic examples but also in the deterministic case, by proving not the existence of a $C^{1}$ unstable manifold, but of a Lipschitz manifold in a neighbourhood of the origin. The theory of inertial manifolds, introduced by Foias et al. (1988) in the deterministic case, and developed by various authors for stochastic equations (Bensoussan \& Flandoli 1995; Chueshov \& Girya 1994), is well suited to this, and we follow some ideas from a paper of Da Prato \& Debussche (1996) in our proof.

In this way, we prove the following theorem. 
Theorem 4.1. If $\lambda_{n}<\beta<\lambda_{n+1}$, then there exists a set $\mathcal{M}_{\delta}(\omega)$, a (locally) invariant n-dimensional Lipschitz manifold, which is part of the unstable set of the origin, i.e.

$$
\lim _{t \rightarrow \infty} \operatorname{dist}(\varphi(-t, \omega) u, 0)=0
$$

for all $u \in \mathcal{M}(\omega)$.

In (4.1), $\varphi(-t, \omega) u$ is the point $v \in \mathcal{M}\left(\theta_{-t} \omega\right)$ such that $\varphi\left(t, \theta_{-t} \omega\right) v=u$ (the fact that $\mathcal{M}(\omega)$ is a finite-dimensional invariant manifold ensures that such a point exists; see proposition 4.6 for more details).

We now appeal to a result of Crauel (2001), which guarantees that the unstable set of the origin (precisely defined as in (4.1)) must be a subset of the random attractor, and so obtain the lower bound on the attractor dimension given in the following theorem.

Theorem 4.2. Provided that $m \leqslant 5$, if $\lambda_{n}<\beta<\lambda_{n+1}$, then $d_{\mathrm{H}}(\mathcal{A}(\omega)) \geqslant n$. In particular, the dimension of the attractor satisfies $d_{\mathrm{H}}(\mathcal{A}(\omega))=O\left(\beta^{m / 2}\right)$.

\section{(a) Truncating the equation}

We prove the existence of $\mathcal{M}_{\delta}(\omega)$ in a series of propositions. We apply a version of the theory in Da Prato \& Debussche (1996) developed to prove the existence of inertial manifolds for stochastic PDEs with a general multiplicative noise term. However, here, we do not look for an inertial manifold, but, in fact, an unstable manifold in a neighbourhood of the origin.

The first task is to truncate the equation in a neighbourhood of the origin, to ensure that the nonlinear term is globally Lipschitz, with a small Lipschitz constant. (Our approach is, in fact, similar to that of Boxler (1991) for centre manifolds, although here, we can truncate our equation in a manner which is independent of $\omega$.) Although this is a standard approach in the deterministic theory, in which one has a compact absorbing ball, it is perhaps the main weakness of the theory of inertial manifolds for stochastic equations, where, in general, the radius of the absorbing ball will depend on $\omega$, and, as yet, there is no proof which allows the Lipschitz constant of the nonlinearity to vary in this way. However, since we require only a local unstable manifold, we will be able to truncate the equation in a consistent fashion.

We are restricted to the case $m \leqslant 5$, for which we can use the embedding $H^{2 \alpha} \subset L^{6}$ with $\frac{3}{4}<\alpha<1$ to show that $F(u)=u^{3}$ is Lipschitz from $H^{2 \alpha}$ into $L^{2}$ (we suppress the $D$ in all our function spaces from now on),

$$
\left|u^{3}-v^{3}\right|_{L^{2}} \leqslant C\left(\|u\|_{H^{2 \alpha}}^{2}+\|v\|_{H^{2 \alpha}}^{2}\right)\|u-v\|_{H^{2 \alpha}} .
$$

Indeed, we have

$$
\begin{aligned}
\left|u^{3}-v^{3}\right|_{L^{2}}^{2} & =\int_{D}\left(u^{3}(x)-v^{3}(x)\right)^{2} \mathrm{~d} x \\
& =\int_{D}\left(\int_{v(x)}^{u(x)} 3 s^{2} \mathrm{~d} s\right)^{2} \mathrm{~d} x \\
& \leqslant 9 \int_{D}|u(x)-v(x)|^{2}\left(u(x)^{2}+v(x)^{2}\right)^{2} \mathrm{~d} x
\end{aligned}
$$

Proc. R. Soc. Lond. A (2001) 


$$
\begin{aligned}
& \leqslant 9\left(\int_{D}|u(x)-v(x)|^{2 p} \mathrm{~d} x\right)^{1 / p}\left(\int_{D}\left|u(x)^{2}+v(x)^{2}\right|^{2 q} \mathrm{~d} x\right)^{1 / q} \\
& \leqslant 9|u-v|_{L^{2 p}}^{2}\left(|u|_{L^{4 q}}+|v|_{L^{4 q}}\right)^{4} \\
& \leqslant 9|u-v|_{L^{6}}^{2}\left(|u|_{L^{6}}+|v|_{L^{6}}\right)^{4},
\end{aligned}
$$

taking $(p, q)=\left(3, \frac{3}{2}\right)$.

For a $C^{1}$ cut-off function $\theta: \mathbb{R}^{+} \rightarrow[0,1]$, such that

$$
\theta(r)= \begin{cases}1, & r \leqslant 1 \\ 0, & r \geqslant 2\end{cases}
$$

with $\left|\theta^{\prime}(r)\right| \leqslant 2$, it is straightforward to verify (see Temam 1988) that

$$
F(u)=-\theta\left(\frac{\|u\|_{H^{2 \alpha}}}{R}\right) u^{3}
$$

is globally bounded,

$$
|F(u)| \leqslant M_{0} \quad \text { for all } u \in H^{2 \alpha},
$$

and globally Lipschitz,

$$
|F(u)-F(v)| \leqslant \tilde{L}\|u-v\|_{H^{2 \alpha}} \text { for all } u, v \in H^{2 \alpha} .
$$

with $\tilde{L} \leqslant c_{0} R^{2}$ for a constant $c_{0}$. Note in particular that $F(u)=-u^{3}$ in $B_{H^{2 \alpha}}(0, R)$, and so the two equations agree in a small $H^{2 \alpha}$-neighbourhood of the origin.

We will find it more convenient to use the norm equivalence between the $H^{2 \alpha}$ norm and the norm in $D\left(A^{\alpha}\right)$. Writing $\left|A^{\alpha} u\right|=|u|_{\alpha}$, where $A$ is the negative Laplacian on $D$ with Dirichlet boundary conditions, we have

$$
\|u\|_{H^{2 \alpha}} \leqslant c|u|_{\alpha}
$$

We can rewrite (4.4) as

$$
|F(u)-F(v)| \leqslant L|u-v|_{\alpha} \quad \text { for all } u, v \in D\left(A^{\alpha}\right),
$$

where $L=c \tilde{L}$ (with $c$ as in (4.5)).

Note that we can make $L$ as small as we wish provided that $R$ in (4.3) is chosen small enough.

(b) An invariant manifold for the truncated equation

We now work with the truncated equation

$$
\mathrm{d} u=(-A u+\beta u+F(u)) \mathrm{d} t+\sigma u \circ \mathrm{d} W_{t},
$$

and prove the existence of an invariant manifold of dimension $n$ when $\lambda_{n}<\beta<\lambda_{n+1}$. In the statement of the theorem we write $P_{n}$ for the orthogonal projection onto the first $n$ eigenfunctions of $A$, ordered so that the corresponding eigenvalues are nondecreasing in $n$, and $Q_{n}$ for its orthogonal complement, $Q_{n}=I-P_{n}$. 
Theorem 4.3. If $\lambda_{n}<\beta<\lambda_{n+1}$, and $R$ in (4.3) is chosen small enough that $L$ satisfies

$$
\beta-\lambda_{n}>4 L \lambda_{n}^{\alpha}
$$

and

$$
\lambda_{n+1}-\beta>(2 K L)^{1 /(1-\alpha)},
$$

then (4.7) has an $n$-dimensional invariant manifold $\mathcal{M}(\omega)$,

$$
\varphi(\lambda, \omega) \mathcal{M}(\omega)=\mathcal{M}\left(\theta_{\lambda} \omega\right), \quad \lambda \geqslant 0 .
$$

Furthermore, $\mathcal{M}(\omega)$ is given as the graph of a Lipschitz function $\Phi_{\omega}: P_{n} H \rightarrow$ $Q_{n} H \cap D\left(A^{\alpha}\right)$,

$$
\left|\Phi_{\omega}\left(p_{1}\right)-\Phi_{\omega}\left(p_{2}\right)\right|_{\alpha} \leqslant 2\left|p_{1}-p_{2}\right|_{\alpha} .
$$

We analyse the equation $\omega$ by $\omega$. To this end, we consider not equation (2.2) for $u$, but, setting $v=\mathrm{e}^{-\sigma W_{t}} u$ and observing that

$$
\mathrm{d} v=\mathrm{e}^{-\sigma W_{t}} \mathrm{~d} u-\mathrm{e}^{-\sigma W_{t}} \sigma u \circ \mathrm{d} W_{t},
$$

we can treat the non-autonomous equation for $v$,

$$
\frac{\mathrm{d} v}{\mathrm{~d} t}=-A v+\beta v+\mathrm{e}^{-\sigma W_{t}} F\left(\mathrm{e}^{\sigma W_{t}} v\right) .
$$

We write this as

$$
\frac{\mathrm{d} v}{\mathrm{~d} t}=-A v+\beta v+F_{\sigma}(v)
$$

where

$$
F_{\sigma}(v)=\mathrm{e}^{-\sigma W_{t}} F\left(\mathrm{e}^{\sigma W_{t}} v\right) .
$$

Note that

$$
\begin{aligned}
\left|F_{\sigma}(u)-F_{\sigma}(v)\right| & \leqslant \mathrm{e}^{-\sigma W_{t}} \mid F\left(\mathrm{e}^{\sigma W_{t}} u\right)-F\left(\mathrm{e}^{\sigma W_{t}} v \mid\right. \\
& \leqslant \mathrm{e}^{-\sigma W_{t}} L\left|\mathrm{e}^{\sigma W_{t}} u-\mathrm{e}^{\sigma W_{t}} v\right|_{\alpha} \\
& =L|u-v|_{\alpha},
\end{aligned}
$$

so that the Lipschitz property of $F$ in (4.6) is transferred to $F_{\sigma}$. Furthermore, it follows, since the support of $F$ is contained in a bounded set in $D\left(A^{\alpha}\right)$, and $F(0)=0$, that

$$
\left|F_{\sigma}(u)\right| \leqslant M_{1}
$$

for some constant $M_{1}$.

We write the solution operator corresponding to the transformed equation as $\psi(t, \omega)$. Clearly,

$$
\psi(t, \omega)=\mathrm{e}^{-\sigma W_{t}(\omega)} \varphi(t, \omega) .
$$

For a function

$$
y:(-\infty, 0] \rightarrow P_{n} H,
$$

we define a norm

$$
\|y\|_{E}=\sup _{t \in(-\infty, 0]}|y(t)|_{\alpha},
$$


and similarly for

$$
z:(-\infty, 0] \rightarrow Q_{n} D\left(A^{\alpha}\right)
$$

For a pair $(y, z)$, we define

$$
\|(y, z)\|_{E}=\max \left(\|y\|_{E},\|z\|_{E}\right) .
$$

The space of all functions $(y, z)$, as in (4.14) and (4.16), with finite $E$ norm (the norm in (4.17)) we denote by $E$.

We will need the following standard bound on the operator norm of $A^{\alpha} \mathrm{e}^{-A t} Q_{n}$ from $L^{2}$ into $L^{2}$ (see, for example, Temam 1988),

$$
\left\|A^{\alpha} \mathrm{e}^{-A t} Q_{n}\right\|_{\text {op }} \leqslant \tilde{K}\left(t^{-\alpha}+\lambda_{n+1}^{\alpha}\right) \mathrm{e}^{-\lambda_{n+1} t} \quad \text { for all } t \geqslant 0 .
$$

We also set

$$
K=\tilde{K} \int_{0}^{\infty}\left(t^{-\alpha}+1\right) \mathrm{e}^{-t} \mathrm{~d} t .
$$

From now on, we write $P=P_{n}$ and $Q=Q_{n}$.

Roughly speaking, we follow the proof in Da Prato \& Debussche (1996). However, here the argument is somewhat different, since our particular choice of multiplicative noise allows us to consider the transformed equation (4.11) $\omega$ by $\omega$, and we do not have to deal with the problems which arise when trying to solve a more general stochastic equation backwards in time. We also need to tailor the analysis to make sure that the dependence on $\beta$ remains explicit.

Proposition 4.4. For each $\omega \in \Omega$, there exists a unique solution $(y, z)$ of the coupled equations

$$
\left.\begin{array}{lll}
\frac{\mathrm{d} y}{\mathrm{~d} t}=-A y+\beta y+P F_{\sigma}(y+z), & y(0)=y_{0} & \\
\frac{\mathrm{d} z}{\mathrm{~d} t}=-A z+\beta z+Q F_{\sigma}(y+z), & z(t) \rightarrow 0 & \text { as } t \rightarrow-\infty,
\end{array}\right\}
$$

on the interval $(-\infty, 0]$.

Proof. We use a contraction mapping argument. For a given pair $(\eta, \xi) \in E$, we define

$$
\mathcal{T}_{y_{0}}(\eta, \xi)=(y, z)
$$

where $(y, z)$ solve the coupled system

$$
\left.\begin{array}{lll}
\frac{\mathrm{d} y}{\mathrm{~d} t}=-A y+\beta y+P F_{\sigma}(\eta+\xi), & y(0)=y_{0} & \\
\frac{\mathrm{d} z}{\mathrm{~d} t}=-A z+\beta z+Q F_{\sigma}(\eta+\xi), & z(t) \rightarrow 0 & \text { as } t \rightarrow-\infty .
\end{array}\right\}
$$

Note that such a solution is well defined, since, using the variation of constants formula on the second equation, we have

$$
z(t)=\mathrm{e}^{-(A-\beta I)(t-\tau)} z(\tau)+\int_{\tau}^{t} \mathrm{e}^{-A(t-s)} Q F_{\sigma}(\xi(s)+\eta(s)) \mathrm{d} s .
$$


Since $(A-\beta I)$ is a positive operator, letting $\tau \rightarrow-\infty$ and using the condition that $z(\tau) \rightarrow 0$, we obtain the unique solution

$$
z(t)=\int_{-\infty}^{t} \mathrm{e}^{-A(t-s)} Q F_{\sigma}(\xi(s)+\eta(s)) \mathrm{d} s .
$$

To show that $\mathcal{T}_{y_{0}}$ is a contraction on $E$, first we estimate the difference between two solutions to the equation

$$
\frac{\mathrm{d} y_{i}}{\mathrm{~d} t}=-A y_{i}+\beta y_{i}+P F_{\sigma}\left(\eta_{i}+\xi_{i}\right), \quad y_{i}(0)=y_{0},
$$

corresponding to two different choices of $(\eta, \xi) \in E$ in (4.21). Writing $y(t)=y_{1}(t)-$ $y_{2}(t)$, it follows that $y$ satisfies

$$
\frac{\mathrm{d} y}{\mathrm{~d} t}=-A y+\beta y+P F_{\sigma}\left(\eta_{1}+\xi_{1}\right)-P F_{\sigma}\left(\eta_{2}+\xi_{2}\right), \quad y(0)=0 .
$$

Using the variation of constants formula, we can write, for $t<0$,

$y(0)=\mathrm{e}^{(A-\beta I) t} y(t)+\int_{t}^{0} \mathrm{e}^{(A-\beta I)(t-s)}\left(P F_{\sigma}\left(\eta_{1}(s)+\xi_{1}(s)\right)-P F_{\sigma}\left(\eta_{2}(s)+\xi_{2}(s)\right)\right) \mathrm{d} s$, and so, since $y(0)=0$,

$$
y(t)=-\int_{t}^{0} \mathrm{e}^{-(A-\beta I) s}\left(P F_{\sigma}\left(\eta_{1}(s)+\xi_{1}(s)\right)-P F_{\sigma}\left(\eta_{2}(s)+\xi_{2}(s)\right)\right) \mathrm{d} s .
$$

Taking the norm in $D\left(A^{\alpha}\right)$ throughout (4.25), we obtain, since $-(A-\beta I)$ is a positive operator on $P H$, with eigenvalues bounded below by $\beta-\lambda_{n}$,

$$
|y(t)|_{\alpha} \leqslant L \lambda_{n}^{\alpha} \int_{t}^{0} \mathrm{e}^{\left(\beta-\lambda_{n}\right) s}\left(|\eta(s)|_{\alpha}+|\xi(s)|_{\alpha}\right) \mathrm{d} s,
$$

where $\eta=\eta_{1}-\eta_{2}$, and similarly for $\xi$.

This becomes

$$
\begin{aligned}
|y(t)|_{\alpha} & \leqslant 2 L \lambda_{n}^{\alpha} \int_{t}^{0} \mathrm{e}^{\left(\beta-\lambda_{n}\right) s}\|(\eta, \xi)\|_{E} \mathrm{~d} s \\
& \leqslant \frac{2 L \lambda_{n}^{\alpha}}{\beta-\lambda_{n}}\|(\eta, \xi)\|_{E}\left[1-\mathrm{e}^{\left(\beta-\lambda_{n}\right) t}\right] .
\end{aligned}
$$

Since $\beta>\lambda_{n}$,

$$
\|y\|_{E} \leqslant \frac{2 L \lambda_{n}^{\alpha}}{\beta-\lambda_{n}}\|(\eta, \xi)\|_{E} .
$$

Equation (4.8) now guarantees that $\|y\|_{E} \leqslant \frac{1}{2}\|(\eta, \xi)\|_{E}$.

For a similar choice of $\left(\eta_{i}, \xi_{i}\right)$, we now estimate the difference between solutions to the two equations

$$
\frac{\mathrm{d} z_{i}}{\mathrm{~d} t}=-A z_{i}+\beta z_{i}+Q F_{\sigma}\left(\eta_{i}+\xi_{i}\right), \quad z_{i}(t) \rightarrow 0 \quad \text { as } t \rightarrow-\infty .
$$

Setting $z=z_{1}-z_{2}$, we have

$$
\frac{\mathrm{d} z}{\mathrm{~d} t}=-A z+\beta z+Q F_{\sigma}\left(\eta_{1}+\xi_{1}\right)-Q F_{\sigma}\left(\eta_{2}+\xi_{2}\right) .
$$


As before, we use the variation of constants formula, and, since $z(t) \rightarrow 0$ as $t \rightarrow-\infty$, we have

$$
z(t)=\int_{-\infty}^{t} \mathrm{e}^{-(A-\beta I)(t-s)} Q\left[F_{\sigma}\left(\eta_{1}+\xi_{1}\right)-F_{\sigma}\left(\eta_{2}+\xi_{2}\right)\right] \mathrm{d} s .
$$

Once more, taking the norm in $D\left(A^{\alpha}\right)$, using the Lipschitz property of $F_{\sigma}$ in $(4.12)$, and the bound on $A^{\alpha} \mathrm{e}^{-A(t-s)} Q$ in (4.18), we have

$$
|z(t)|_{\alpha} \leqslant \tilde{K} L \int_{-\infty}^{t}\left((t-s)^{-\alpha}+\left(\lambda_{n+1}-\beta\right)^{\alpha}\right) \mathrm{e}^{-\left(\lambda_{n+1}-\beta\right)(t-s)}\left(|\eta(s)|_{\alpha}+|\xi(s)|_{\alpha}\right) \mathrm{d} s .
$$

Using the definition of the norm in $E$ (4.17) now gives

$$
\begin{aligned}
|z(t)|_{\alpha} & \leqslant \tilde{K} L \int_{-\infty}^{t}\left((t-s)^{-\alpha}+\left(\lambda_{n+1}-\beta\right)^{\alpha}\right) \mathrm{e}^{-\left(\lambda_{n+1}-\beta\right)(t-s)}\|(\eta, \xi)\|_{E} \mathrm{~d} s \\
& \leqslant \tilde{K} L\|(\eta, \xi)\|_{E} \int_{-\infty}^{0}\left(|\tau|^{-\alpha}+\left(\lambda_{n+1}-\beta\right)^{\alpha}\right) \mathrm{e}^{\left(\lambda_{n+1}-\beta\right) \tau} \mathrm{d} \tau \\
& \leqslant \tilde{K} L\|(\eta, \xi)\|_{E} \int_{-\infty}^{0}\left(|\tau|^{-\alpha}+\left(\lambda_{n+1}-\beta\right)^{\alpha}\right) \mathrm{e}^{\left(\lambda_{n+1}-\beta\right) \tau} \mathrm{d} \tau \\
& \leqslant \frac{\tilde{K} L}{\lambda_{n+1}-\beta}\|(\eta, \xi)\|_{E}\left(\lambda_{n+1}-\beta\right)^{\alpha} \int_{-\infty}^{0}\left[|u|^{-\alpha}+1\right] \mathrm{e}^{u} \mathrm{~d} u .
\end{aligned}
$$

It follows that

$$
\|z\|_{E} \leqslant \frac{K L}{\left(\lambda_{n+1}-\beta\right)^{1-\alpha}}\|(\eta, \xi)\|_{E},
$$

where the constant $K$ is defined in (4.19).

The condition on $L$ in (4.9) now ensures that $\|z\|_{E} \leqslant \frac{1}{2}\|(\eta, \xi)\|_{E}$. It follows that $\|(y, z)\|_{E} \leqslant \frac{1}{2}\|(\eta, \xi)\|_{E}$, and so $\mathcal{T}_{y_{0}}$ is a contraction, and we obtain the unique solution to our coupled pair of equations.

We now define

$$
\Phi_{\omega}\left(y_{0}\right)=z(0),
$$

where $z$ is the solution to $(4.20)$ with $y(0)=y_{0}$ and noise $W_{t}(\omega)$. Observe in particular that $\Phi_{\omega}(0)=0$ for all $\omega \in \Omega$.

Proposition 4.5. For almost every $\omega \in \Omega$,

$$
\left|\Phi_{\omega}\left(y_{1}^{0}\right)-\Phi_{\omega}\left(y_{2}^{0}\right)\right|_{\alpha} \leqslant 2\left|y_{1}^{0}-y_{2}^{0}\right|_{\alpha},
$$

and the graph $\mathcal{M}(\omega)$ of $\Phi_{\omega}$ is invariant for $\varphi$,

$$
\varphi(\lambda, \omega) \mathcal{M}(\omega)=\mathcal{M}\left(\theta_{\lambda} \omega\right), \quad \lambda \geqslant 0,
$$

i.e. for any $y_{0} \in P H$ and $\lambda \geqslant 0$, we have

$$
\varphi(\lambda, \omega)\left(y_{0}+\Phi_{\omega}\left(y_{0}\right)\right)=y_{\lambda}+\Phi_{\theta_{\lambda} \omega}\left(y_{\lambda}\right),
$$

P-a.s., where

$$
y_{\lambda}=P \varphi(\lambda, \omega)\left(y_{0}+\Phi_{\omega}\left(y_{0}\right)\right) .
$$


Proof. First we show the Lipschitz property of $\Phi_{\omega}$ by estimating

$$
\left\|\mathcal{T}_{y_{0}^{1}}(\eta, \xi)-\mathcal{T}_{y_{0}^{2}}(\eta, \xi)\right\|_{E}
$$

We will write

$$
\left(y^{i}, z^{i}\right)=\mathcal{T}_{y_{i}^{0}}(\eta, \xi) .
$$

Since $z^{1}=z^{2}$, we need only estimate the difference $y=y_{1}-y_{2}$ between two solutions of

$$
\frac{\mathrm{d} y_{i}}{\mathrm{~d} t}=-(A-\beta I) y_{i}+P F_{\sigma}(\eta+\xi), \quad y_{i}(0)=y_{0}^{i},
$$

but with the same pair $(\eta, \xi) \in E$. So,

$$
\frac{\mathrm{d} y}{\mathrm{~d} t}=-(A-\beta I) y .
$$

Thus,

$$
y(0)=\mathrm{e}^{(A-\beta I) t} y(t)
$$

and so, for $t<0$,

$$
|y(t)|_{\alpha} \leqslant \mathrm{e}^{\left(\beta-\lambda_{n}\right) t}\left|y_{0}^{1}-y_{0}^{2}\right|_{\alpha} .
$$

It follows, since $\beta>\lambda_{n}$, that

$$
\left\|\mathcal{T}_{y_{0}^{1}}(\eta, \xi)-\mathcal{T}_{y_{0}^{2}}(\eta, \xi)\right\|_{E} \leqslant\left|y_{0}^{1}-y_{0}^{2}\right|_{\alpha} .
$$

So, we have

$$
\begin{aligned}
\left\|\left(y^{1}, z^{1}\right)-\left(y^{2}, z^{2}\right)\right\|_{E} & =\left\|\mathcal{T}_{y_{0}^{1}}\left(y^{1}, z^{1}\right)-\mathcal{T}_{y_{0}^{2}}\left(y^{2}, z^{2}\right)\right\|_{E} \\
& \leqslant\left\|\mathcal{T}_{y_{0}^{1}}\left(y^{1}, z^{1}\right)-\mathcal{T}_{y_{0}^{2}}\left(y^{1}, z^{1}\right)\right\|_{E}+\left\|\mathcal{T}_{y_{0}^{2}}\left(y^{1}, z^{1}\right)-\mathcal{T}_{y_{0}^{2}}\left(y^{2}, z^{2}\right)\right\|_{E} \\
& \leqslant\left|y_{0}^{1}-y_{0}^{2}\right|_{\alpha}+\frac{1}{2}\left\|\left(y^{1}, z^{1}\right)-\left(y^{2}, z^{2}\right)\right\|_{E}
\end{aligned}
$$

and so

$$
\left\|\left(y^{1}, z^{1}\right)-\left(y^{2}, z^{2}\right)\right\|_{E} \leqslant 2\left|y_{0}^{1}-y_{0}^{2}\right|_{\alpha} .
$$

Since $\Phi_{\omega}\left(y_{0}^{i}\right)=z^{i}(0)$, the Lipschitz bound follows.

To show the invariance, observe that $\Phi_{\omega}\left(y_{0}\right)$ is defined as the solution at time 0 of the $z$ component of (4.20) when $y(0)=y_{0}$. Solving (4.11) with the initial condition $y_{0}+\Phi_{\omega}\left(y_{0}\right)$ produces a solution to the coupled equations

$$
\begin{array}{lll}
\frac{\mathrm{d} y}{\mathrm{~d} t}=-A y+\beta y+P F_{\sigma}(y+z), & y(\lambda) & =P \psi(\lambda, \omega)\left[y_{0}+\Phi_{\omega}\left(y_{0}\right)\right] \\
\frac{\mathrm{d} z}{\mathrm{~d} t}=-A z+\beta z+Q F_{\sigma}(y+z), & z(t) \rightarrow 0 & \text { as } t \rightarrow-\infty,
\end{array}
$$

which is unique, by proposition 4.4. Now, observe that $z(\lambda)$ is the same as the $z(0)$ produced as the solution to (4.11) with $W_{t}(\omega)$ replaced by

$$
W_{t+\lambda}(\omega)=W_{t}\left(\theta_{\lambda} \omega\right)+W_{\lambda}(\omega) .
$$

With this change, we obtain the solution to (4.11) with $\omega$ replaced by $\theta_{\lambda} \omega$, but multiplied by an additional factor of $\mathrm{e}^{\sigma W_{\lambda}(\omega)}$.

Therefore, we do not obtain an invariance of $\mathcal{M}(\omega)$ for the transformed equation, but rather

$$
\psi(\lambda, \omega) \mathcal{M}(\omega)=\mathrm{e}^{\sigma W_{\lambda}(\omega)} \mathcal{M}\left(\theta_{\lambda} \omega\right) .
$$

The required invariance property for the untransformed equation is now immediate using (4.13). 
(c) A local unstable manifold for the original equation

Finally, we show that the inertial manifold found in the previous section is, in fact, part of the unstable set of the origin, within the small neighbourhood on which the truncated and original equations agree.

Proposition 4.6. There exists a $\delta(\omega)$ such that $\mathcal{M}_{\delta}(\omega)$, the intersection of $\mathcal{M}(\omega)$ with the ball $B_{H^{2 \alpha}}(0, \delta(\omega))$, is part of the unstable set of the origin for the original equation.

Proof. We consider the stochastic 'inertial form', i.e. the equation on the inertial manifold for the $P$ components of $u$, and show that there exists a $\delta(\omega)<R$ such that, for all $p_{0}$ with $\left|p_{0}\right|_{\alpha} \leqslant \delta$,

(i) $|p(t)|_{\alpha} \leqslant R$ for all $t \leqslant 0$, and

(ii) $p(t) \rightarrow 0$ as $t \rightarrow-\infty$.

Point (i) shows that the trajectory 'backward' from $p_{0}+\Phi_{\omega}\left(p_{0}\right)$ remains within $B_{H^{2 \alpha}}(0, R)$, so that it is, in fact, a solution to the original (untruncated) equation, while (ii) shows that $p_{0}+\Phi_{\omega}\left(p_{0}\right)$ is an element of the unstable set of the origin.

Note that we can consider trajectories 'backwards in time', since $\mathcal{M}(\omega)$ is invariant: $\varphi\left(t, \theta_{-t} \omega\right) \mathcal{M}\left(\theta_{-t} \omega\right)=\mathcal{M}(\omega)$, and so for $u \in \mathcal{M}(\omega)$ there exists at least one point $v$ in $\mathcal{M}\left(\theta_{-t} \omega\right)$ such that $\varphi\left(t, \theta_{-t} \omega\right) v=u$. Since the governing equation reduces to a finite-dimensional random ordinary differential equation on $\mathcal{M}(\omega)$ (see below), this point is unique.

The inertial form is

$$
\mathrm{d} p=\left\{-(A-\beta I) p+P F\left(p+\Phi_{\theta_{t} \omega}(p)\right)\right\} \mathrm{d} t+\sigma p \circ \mathrm{d} W_{t} .
$$

Writing $x(t)=\mathrm{e}^{-\sigma W_{t}} p(t)$, this becomes

$$
\frac{\mathrm{d} x}{\mathrm{~d} t}=-(A-\beta I) x+\mathrm{e}^{-\sigma W_{t}} P F\left(\mathrm{e}^{\sigma W_{t}} x+\Phi_{\theta_{t} \omega}\left(\mathrm{e}^{\sigma W_{t}} x\right)\right) .
$$

Now, we have

$$
\left|A^{\alpha} P F(u)\right| \leqslant \lambda_{n}^{\alpha}|F(u)| \leqslant L \lambda_{n}^{\alpha}|u|_{\alpha},
$$

since $F$ is Lipschitz and $F(0)=0$. Therefore, we have, noting that the Lipschitz constant of $\Phi_{\omega}$ in (4.10) is independent of $\omega$,

$$
\frac{1}{2} \frac{\mathrm{d}}{\mathrm{d} t}|x(-t)|_{\alpha}^{2} \leqslant-\left(\beta-\lambda_{n}\right)|x(-t)|_{\alpha}^{2}+\mathrm{e}^{-\sigma W_{-t}} \lambda_{n}^{\alpha} 3 L \mathrm{e}^{\sigma W_{-t}}|x(-t)|_{\alpha}^{2},
$$

or

$$
\frac{\mathrm{d}}{\mathrm{d} t}|x(-t)|_{\alpha} \leqslant-\left(\beta-\lambda_{n}-3 \lambda_{n}^{\alpha} L\right)|x(-t)|_{\alpha} .
$$

Note that it follows from (4.8) that

$$
\gamma \equiv \beta-\lambda_{n}-3 \lambda_{n}^{\alpha} L>\lambda_{n}^{\alpha} L,
$$

and in particular is positive. We therefore have, for $t<0$,

$$
|x(t)|_{\alpha} \leqslant \mathrm{e}^{\gamma t}\left|x_{0}\right|_{\alpha},
$$


which gives, for $p(t)$,

$$
|p(t)|_{\alpha} \leqslant \mathrm{e}^{\gamma t} \mathrm{e}^{\sigma W_{t}}\left|p_{0}\right|_{\alpha}
$$

Now, for each fixed $\omega$, given $\epsilon>0$ there exists a constant $C_{\epsilon}(\omega)$ such that

$$
\mathrm{e}^{\sigma W_{t}} \leqslant C_{\epsilon} \mathrm{e}^{-\epsilon t} \quad \text { for all } t \leqslant 0 .
$$

Therefore, we have

$$
|p(t)|_{\alpha} \leqslant \mathrm{e}^{\gamma t} \mathrm{e}^{-\epsilon t} C_{\epsilon}(\omega)\left|p_{0}\right|_{\alpha},
$$

and so, if we choose $\delta(\omega)=\min \left(R, R / C_{\epsilon}(\omega)\right)$, the result follows.

\section{A bifurcation of pitchfork type}

We now make further use of the unstable manifold to help us prove the existence of a stochastic bifurcation as $\beta$ increases through $\lambda_{1}$.

\section{(a) A definition of a stochastic pitchfork bifurcation}

To motivate our definition, we briefly recall a simple example in which such a bifurcation has been studied by Arnold \& Boxler (1992).

The stochastic ordinary differential equation

$$
\mathrm{d} x=\left(\beta x-x^{3}\right) \mathrm{d} t+\sigma x \circ \mathrm{d} W_{t},
$$

can be solved explicitly. As $\beta$ passes through zero from below, behaviour similar to that in the deterministic case can be observed. A random fixed point is a random set which, for almost all $\omega$, consists of just one point, $a(\omega)$, and is invariant,

$$
\varphi(t, \omega) a(\omega)=a\left(\theta_{t} \omega\right) .
$$

Arnold \& Boxler (1992) show that for $\beta<0$ the random attractor is just the fixed point $\{0\}$, whereas for $\beta>0$, two new random fixed points appear, $\pm a(\omega)$. The random attractor now consists of the interval $[-a(\omega),+a(\omega)]$.

Crauel et al. (1999) discuss such bifurcations in one-dimensional systems in more detail, and give a definition of a stochastic pitchfork bifurcation in terms of random invariant measures. They define a stochastic pitchfork bifurcation as the appearance of two new invariant measures, associated with new random fixed points, which are stable in an appropriate sense.

Below, we adopt a definition of a pitchfork bifurcation based on the appearance of new random fixed points rather than in terms of invariant measures.

Definition 5.1. Let $\left(\varphi_{\beta}\right)_{\beta \in \mathbb{R}}$ be a family of RDS parametrized by $\beta$. We say that $\left(\varphi_{\beta}\right)$ undergoes a stochastic pitchfork bifurcation at $\beta=\beta_{0}$ if

(i) for $\beta \leqslant \beta_{0}$, the random attractor consists solely of one (random) fixed point, $\{0\}$, and

(ii) for $\beta>\beta_{0}$, the random attractor contains $\{0\}$ and two new random fixed points, $a^{+}(\omega)$ and $a^{-}(\omega)$, and $\mathbb{P}$-a.s. $a^{ \pm}(\omega) \rightarrow 0$ as $\beta \downarrow \beta_{0}$. 
Although, unlike Crauel et al. (1999), we do not include a criterion of stability for the new fixed points, we believe that this type of bifurcation still merits the title 'pitchfork', and we now prove that, in the case of a one-dimensional domain, such a bifurcation occurs as $\beta$ passes through $\lambda_{1}$. Some discussion of the stability of the new fixed points is given at the end of $\S 5$.

We showed in a previous paper (Caraballo et al. 2000) that, for $\beta<\lambda_{1}$, the random attractor consists solely of the fixed point $u \equiv 0$. To study the structure of the attractor for $\beta>\lambda_{1}$, we argue as follows. First we show that the manifold $\mathcal{M}(\omega)$ is tangent to the linear space spanned by the first eigenfunction of the Laplacian, and, with a little care, it follows that this manifold intersects the cone $\mathcal{K}^{+}$of positive functions. Since $\mathcal{K}^{+}$is in fact invariant for the cocycle (see, for example, Kotelenez 1992), the restriction of the cocycle to $\mathcal{K}^{+}$has a non-trivial attractor $\mathcal{A}^{+}(\omega)$. Now, since the cocycle is order-preserving, the theory developed by Arnold \& Chueshov (1998) shows that functions in $\mathcal{A}^{+}(\omega)$ are bounded above and below by random fixed points: below by zero, and above by a new random fixed point $a^{+}(\omega)$. The same argument applies in $\mathcal{K}^{-}$and proves the presence of a pitchfork bifurcation.

\section{(b) Differentiability of $\mathcal{M}(\omega)$ at 0}

First we prove the differentiability property of the manifold. In particular, the cubic bound (5.1) will be used to show that a portion of $\mathcal{M}(\omega)$ lies in $\mathcal{K}^{+}$.

Proposition 5.2. The manifold $\mathcal{M}(\omega)$ is tangent to $P_{n} H$ at the origin, and, moreover,

$$
\left|\Phi_{\omega}(p)\right|_{\alpha} \leqslant C_{\omega}|p|_{\alpha}^{3} .
$$

Proof. First observe that

$$
F_{\sigma}(u)=-\mathrm{e}^{-\sigma W_{t}} \theta\left(\frac{\left|u \mathrm{e}^{\sigma W_{t}}\right|_{\alpha}}{R}\right)\left(u \mathrm{e}^{\sigma W_{t}}\right)^{3}
$$

is essentially cubic, so that

$$
\begin{aligned}
\left|F_{\sigma}(u)\right| & \leqslant \mathrm{e}^{2 \sigma W_{t}}\left(\int u^{6} \mathrm{~d} x\right)^{1 / 2} \\
& \leqslant \mathrm{e}^{2 \sigma W_{t}}\|u\|_{L^{6}}^{3} \\
& \leqslant C \mathrm{e}^{2 \sigma W_{t}}|u|_{\alpha}^{3} .
\end{aligned}
$$

Now, $\Phi_{\omega}\left(y_{0}\right)$ is $z(0)$ from the coupled equations in proposition 4.4, so we know (see (4.22)) that

$$
z(0)=\int_{-\infty}^{0} \mathrm{e}^{(A-\beta I) s} Q F_{\sigma}(y(s)+z(s)) \mathrm{d} s,
$$

and hence,

$$
|z(0)|_{\alpha} \leqslant \tilde{K} \int_{-\infty}^{t}\left((-s)^{-\alpha}+\left(\lambda_{n+1}-\beta\right)^{\alpha}\right) \mathrm{e}^{\left(\lambda_{n+1}-\beta\right) s} C \mathrm{e}^{2 \sigma W_{s}}|u|_{\alpha}^{3} \mathrm{~d} s .
$$

Since

$$
|u|_{\alpha}^{3} \leqslant\left(|y|_{\alpha}+|z|_{\alpha}\right)^{3} \leqslant 8\left(|y|_{\alpha}^{3}+|z|_{\alpha}^{3}\right),
$$


we have, using (4.30),

$$
|z(0)|_{\alpha} \leqslant K_{\omega}\left[\|y\|_{E}^{3}+\|z\|_{E}^{3}\right]
$$

Now, we know that

$$
z(t)=\int_{-\infty}^{t} \mathrm{e}^{-(A-\beta I)(t-s)} Q F_{\sigma}(y+z) \mathrm{d} s,
$$

and we can estimate $|z(t)|_{\alpha}$ as in the proof of proposition 4.4, using the fact that $F_{\sigma}(0)=0$,

$$
\begin{aligned}
|z(t)|_{\alpha} & \leqslant \tilde{K} L \int_{-\infty}^{t}\left((t-s)^{\alpha}+\left(\lambda_{n+1}-\beta\right)^{\alpha}\right) \mathrm{e}^{-\left(\lambda_{n+1}-\beta\right)(t-s)}|y+z|_{\alpha} \mathrm{d} s \\
& \left.\leqslant\left(\tilde{K} L \int_{-\infty}^{t}\left((t-s)^{\alpha}+\left(\lambda_{n+1}-\beta\right)^{\alpha}\right)\right) \mathrm{e}^{-\left(\lambda_{n+1}-\beta\right)(t-s)} \mathrm{d} s\right)\left(\|y\|_{E}+\|z\|_{E}\right) .
\end{aligned}
$$

Now, we saw in proposition 4.4 that the integral expression here is bounded by $\frac{1}{2}$, and so, we have $\|z\|_{E} \leqslant \frac{1}{2}\left(\|y\|_{E}+\|z\|_{E}\right)$, i.e. $\|z\|_{E} \leqslant\|y\|_{E}$.

Equation (5.2) now becomes

$$
|z(0)|_{\alpha} \leqslant 2 K_{\omega}\|y\|_{E}^{3},
$$

so it only remains to estimate $\|y\|_{E}$. However, this is the solution to the governing equation which has $y(0)=y_{0}$ and $z(0)=\Phi_{\omega}\left(y_{0}\right)$, and so which lies on $\mathcal{M}(\omega)$. The inequality (4.28) from proposition 4.6 shows that, in fact,

$$
|y(t)|_{\alpha} \leqslant\left|y_{0}\right|_{\alpha} \text { for all } t \leqslant 0,
$$

and so $\|y\|_{E} \leqslant\left|y_{0}\right|_{\alpha}$. Combining this with (5.3) gives the required bound.

\section{(c) The bifurcation theorem}

We now prove our second main result.

Theorem 5.3. Let $m=1$. Then (2.2) undergoes a stochastic pitchfork bifurcation at $\beta=\lambda_{1}$. In particular, for $\beta>\lambda_{1}$ there exist positive and negative random fixed points $\pm a(\omega)$, and $a(\omega) \rightarrow 0$ as $\beta \downarrow \lambda_{1}$.

Note that one could easily recast this result in terms of the appearance of two new invariant measures, namely the random Dirac measures concentrated at $\pm a(\omega)$.

Proof. Choose and fix $\beta>\lambda_{1}$. Since the first eigenfunction of the Laplacian is positive, we use the cubic estimate to show that $\mathcal{M}_{\delta}(\omega)$ must contain a positive function. More precisely, we let

$$
\mathcal{K}^{+}=\left\{u \in L^{2}(D): u(x) \geqslant 0 \text { almost everywhere }\right\},
$$

and

$$
\mathcal{K}^{-}=\left\{u \in L^{2}(D): u(x) \leqslant 0 \text { almost everywhere }\right\} .
$$

The main idea is to show that one portion of the unstable manifold must be a subset of $\mathcal{K}^{+}$, and another a subset of $\mathcal{K}^{-}$. 
On a one-dimensional domain, $[0, L]$, we know that the eigenfunctions of the Laplacian with Dirichlet boundary conditions are proportional to

$$
w_{n}(x)=\sin (n \pi x / L) .
$$

Since the manifold $\mathcal{M}_{\delta}(\omega)$ is given as a graph over the space spanned by the first eigenfunction, for each fixed $\omega$ the manifold consists of a family of functions (here parametrized by $\epsilon$ ) given in the form

$$
u(x, \epsilon)=\epsilon \sin (\pi x / L)+\sum_{j=2}^{\infty} c_{j} \sin (j \pi x / L),
$$

where $c_{j}=c_{j}(\epsilon, \omega)$.

Since

$$
\left|\frac{\sin (j \pi x / L)}{\sin (\pi x / L)}\right| \leqslant j
$$

(simply rewrite the sine terms using complex exponentials), we have

$$
u(x, \epsilon) \geqslant \sin (\pi x / L)\left[\epsilon-\sum_{j=2}^{\infty}\left|c_{j}\right| j\right] .
$$

Using the cubic bound on $\Phi_{\omega}$, we know that

$$
\left|\sum_{j=2}^{\infty} c_{j} \sin (j \pi x / L)\right|_{\alpha}=C\left(\sum_{j=2}^{\infty}\left|c_{j}\right|^{2} j^{4 \alpha}\right)^{1 / 2} \leqslant C_{\omega}|\epsilon \sin (\pi x / L)|_{\alpha}^{3}=K_{\omega} \epsilon^{3} .
$$

Since $\alpha>\frac{3}{4}$, we can now write

$$
\sum_{j=2}^{\infty}\left|c_{j}\right| j \leqslant\left(\sum_{j=2}^{\infty} j^{4 \alpha}\left|c_{j}\right|^{2}\right)^{1 / 2}\left(\sum_{j=2}^{\infty} j^{2(1-2 \alpha)}\right)^{1 / 2} \leqslant C \epsilon^{3}
$$

and so

$$
u(x, \epsilon) \geqslant \sin (\pi x / L)\left[\epsilon-C \epsilon^{3}\right] .
$$

Thus, for $\epsilon>0$ and small enough, $u(x, \epsilon) \geqslant 0$ on $[0, L]$; similarly, for $\epsilon<0$ and small enough, $u(x, \epsilon) \leqslant 0$ on $[0, L]$. In other words, there are portions of $\mathcal{M}_{\delta}(\omega)$ near the origin which intersect non-trivially with $\mathcal{K}^{+}$and $\mathcal{K}^{-}$.

Now, Kotelenez (1992) shows that the cocycle generated by the equation is orderpreserving on $L^{2}(D)$, so that if $u_{0} \geqslant v_{0}$ almost everywhere, then

$$
\varphi(t, \omega) u_{0} \geqslant \varphi(t, \omega) v_{0} .
$$

In particular, since zero is a fixed point of the equation, $\mathcal{K}^{ \pm}$are invariant subspaces for $\varphi$. (These two facts are easy to check, if $u_{0}$ and $v_{0}$ are continuous functions, by applying the standard deterministic theory (essentially the maximum principle, see Smith (1995)) to the equation for $v(t)=\mathrm{e}^{-\sigma W_{t}} u(t)$.)

By following the analysis in Caraballo et al. (2000), one can show that the flow in each of these subspaces has a positively invariant compact absorbing set, namely a bounded set in $H^{1}(D)$ with radius $r(\omega)$, where $r(\omega)$ is a tempered random variable. 
In particular, therefore, using the theory in Flandoli \& Schmalfuss (1996), the flow in each of these subspaces has its own random attractor, $\mathcal{A}^{+}(\omega)$ and $\mathcal{A}^{-}(\omega)$, and these attract all tempered random sets. Since $\mathcal{M}_{\delta}(\omega) \cap \mathcal{K}^{ \pm}$forms part of the unstable set of the origin, it must be a subset of $\mathcal{A}^{ \pm}(\omega)$, and so, in particular, $\mathcal{A}^{ \pm}(\omega)$ is non-trivial.

Arnold \& Chueshov (1998) develop an extensive theory for order-preserving random dynamical systems, and, in particular, one of their results (theorem 2) provides the existence of new stochastic fixed points in this example (essentially, we follow Chueshov (2000)): if $\varphi$ is an order-preserving random dynamical system for which there exists a random interval

$$
\mathcal{J}(\omega)=\{u: b(\omega) \leqslant u \leqslant c(\omega)\}
$$

which is attracted to and contains $\mathcal{A}(\omega)$, then there exist random fixed points $u_{-}(\omega) \leqslant u_{+}(\omega)$ such that

$$
u_{-}(\omega) \leqslant u \leqslant u_{+}(\omega) \text { for all } u \in \mathcal{A}(\omega) .
$$

Since $\mathcal{A}(\omega)$ lies within a random ball in $H^{1}(D)$ of radius $r(\omega)$, and since $H^{1}(D) \subset$ $C^{0}(D)$ with $\|u\|_{\infty} \leqslant C(D)\|u\|_{H}^{1}$, it follows, in particular, that

$$
0 \leqslant u \leqslant c(D) r(\omega) \text { for all } u \in \mathcal{A}^{+}(\omega) .
$$

Using the above result, we can deduce that there exist two random fixed points $u_{-}(\omega)$ and $u_{+}(\omega)$ such that

$$
u_{-}(\omega) \leqslant u \leqslant u_{+}(\omega) \text { for all } u \in \mathcal{A}^{+}(\omega) .
$$

Clearly, $u_{-}(\omega)=0$, but since $\mathcal{A}^{+}(\omega)$ is non-trivial, this proves the existence of a new random (positive) fixed point $a(\omega)=u_{+}(\omega)$. By symmetry, there is also a new random (negative) fixed point $-a(\omega)$. (For a similar argument, see Chueshov (2000).)

That $a(\omega) \rightarrow 0$ as $\beta \downarrow \lambda_{1}$ follows from a slight variant of the general result on the upper-semicontinuity of random attractors to be found in Caraballo \& Langa (2001, theorem 3), which guarantees, under conditions which are easily verified in our case (convergence of the corresponding cocyles; convergence of the compact absorbing sets; and positive invariance of the compact absorbing set for the limit equation $\left.\left(\beta=\lambda_{1}\right)\right)$, that $\mathbb{P}$-a.s.

$$
\lim _{\beta \downarrow \lambda_{1}} \operatorname{dist}\left(\mathcal{A}_{\beta}^{+}(\omega), A_{\lambda_{1}}(\omega)\right)=0 .
$$

Since $a(\omega) \subset \mathcal{A}^{+}(\omega)$, and $\mathcal{A}_{\lambda_{1}}(\omega)=\{0\}$, we have $a(\omega) \rightarrow 0$.

In fact, we know a little more than is stated in the theorem. For $\beta>\lambda_{1}$ the attractor contains the two distinct, non-trivial, invariant subsets $\mathcal{A}^{+}(\omega)$ and $\mathcal{A}^{-}(\omega)$, which attract all initial conditions in $\mathcal{K}^{+}$and $\mathcal{K}^{-}$, respectively. One can think of this, loosely, as a transfer of stability of the origin to $\mathcal{A}^{+}(\omega)$ and $\mathcal{A}^{-}(\omega)$.

For a more concrete stability result, theorem 2 in Arnold \& Chueshov (1998) guarantees a limited type of stability for the new fixed points. In particular, $a(\omega)$ is stable from above, in that if $u(\omega) \geqslant a(\omega)$ (note that this 'initial condition' cannot in general be taken to be deterministic), then

$$
\lim _{t \rightarrow \infty} \varphi\left(t, \theta_{-t} \omega\right) u\left(\theta_{-t} \omega\right)=a(\omega)
$$


(stability 'from below' holds for $-a(\omega)$ ). Since trajectories near zero in $\mathcal{K}^{+}$move away from the origin, it seems reasonable to expect that $a(\omega)$ is attracting in $\mathcal{K}^{+}$. If one could show that solutions eventually enter $\mathcal{K}^{+}$or $\mathcal{K}^{-}$, this would give the stability of the fixed points. (A general result guaranteeing the existence of stable fixed points is given by Schmalfuß (1996), but we have not been able to apply this in our case.)

\section{Conclusion}

We have shown that the structure of the random attractor for our example, the Chafee-Infante equation with a multiplicative noise term, is surprisingly close to that of the deterministic equation. In particular, we have shown that the dimension of the attractor, asymptotically in $\beta$, exhibits the same behaviour as in the deterministic case, and is independent of the level of noise $(\sigma)$.

Analysing further the structure of this attractor, we have shown that as $\beta$ passes through $\lambda_{1}$, the attractor grows and two new random fixed points appear. Noting that $a^{ \pm}(\omega) \in \mathcal{A}^{ \pm}(\omega)$, what we expect is that, in fact, for $\lambda_{1}<\beta<\lambda_{2}$,

$$
\mathcal{A}(\omega)=\mathcal{A}^{+}(\omega) \cup A^{-}(\omega),
$$

with $\mathcal{A}^{ \pm}(\omega)$ consisting of a one-dimensional manifold (the image of $\mathcal{M}_{\delta}(\omega) \cap \mathcal{K}^{ \pm}$ under the flow) joining the origin to $\pm a(\omega)$. This would ensure that the attractor's structure is exactly that in the deterministic case.

Some support for this picture, at least for small $\sigma$, is given by the upper semicontinuity result in Caraballo et al. (1998). We proved there that, as $\sigma \rightarrow 0$, for each $\omega$,

$$
\operatorname{dist}\left(\mathcal{A}_{\sigma}(\omega), \mathcal{A}\right) \rightarrow 0
$$

Thus, the random attractor, a set which is at least one dimensional, must lie within a small neighbourhood of $\mathcal{A}$, which is itself homeomorphic to a line when $\lambda_{1}<\beta<\lambda_{2}$.

Finally, we comment that we would not expect similar ideas to work in the case of an additive noise term (e.g. $+\epsilon \phi \mathrm{d} W_{t}$, for some $\phi \in H$ ). Without the fixed point $\{0\}$, there is no clear way to show that an invariant manifold for a truncated version of the equation is a subset of the random attractor. Indeed, the one-dimensional example in Crauel \& Flandoli (1998) suggests that, with an additive noise, the attractor will be very much simpler than in the deterministic case. We hope to investigate this further in a future paper.

Many thanks to Igor Chueshov, Hans Crauel, Franco Flandoli and Antonio Suárez for some interesting and helpful conversations. J.C.R. is currently a Royal Society University Research Fellow, and thanks the Society for its support. He also thanks EDAN for their hospitality, and Iberdrola for their financial assistance during his visit. T.C. and J.A.L. have been partly supported by DGICYT project PB98-1134.

\section{References}

Arnold, L. 1998 Random dynamical systems. Springer.

Arnold, L. \& Boxler, P. 1992 Stochastic bifurcation: instructive examples in dimension one. In

Diffusion processes and related problems in analysis (ed. N. A. Pinsky \& V. Wihstutz), vol. II, pp. 241-256. Stuttgart: Birkhäuser. 
Arnold, L. \& Chueshov, I. 1998 Order-preserving random dynamical systems: equilibria, attractors, applications. Dynam. Stability Sys. 13, 265-280.

Babin, A. V. \& Vishik, M. I. 1983 Attractors of partial differential evolution equations and estimates of their dimension. Russ. Math. Surv. 38, 151-213.

Bensoussan, A. \& Flandoli, F. 1995 Stochastic inertial manifolds. Stoch. Stoch. Rep. 53, 13-39.

Boxler, P. 1991 How to construct stochastic center manifolds on the level of vector fields. In Lyapunov exponents. Springer Lectures Notes in Mathematics, no. 1486, pp. 141-158. Springer.

Caraballo, T. \& Langa, J. A. 2001 On the upper semicontinuity of cocycle attractors for nonautonomous and random dynamical systems. Dynam. Cont. Discrete Imp. Sys. In the press.

Caraballo, T., Langa, J. A. \& Robinson, J. C. 1998 Upper semicontinuity of attractors for small random perturbations of dynamical systems. Commun. PDEs 23, 1557-1581.

Caraballo, T., Langa, J. A. \& Robinson, J. C. 2000 Stability and random attractors for a reaction-diffusion equation with multiplicative noise. Discrete Cont. Dynam. Sys. 6, 875892.

Chueshov, I. D. 2000 Gevrey regularity of random attractors for stochastic reaction-diffusion equations. Random Op. Stoch. Eqns 8, 143-162.

Chueshov, I. D. \& Girya, T. V. 1994 Inertial manifolds for stochastic dissipative dynamical systems. Dokl. Acad. Sci. Ukraine 7, 42-45.

Crauel, H. 2001 Random point attractors versus random set attractors. J. Lond. Math. Soc. 63, $413-427$.

Crauel, H. \& Flandoli, F. 1994 Attractors for random dynamical systems. Prob. Theory Relat. Fields 100, 365-393.

Crauel, H. \& Flandoli, F. 1998 Additive noise destroys a pitchfork bifurcation. J. Dynam. Diff. Eqns 10, 259-274.

Crauel, H., Debussche, A. \& Flandoli, F. 1997 Random attractors. J. Dynam. Diff. Eqns 9, 307-341.

Crauel, H., Imkeller, P. \& Steinkamp, M. 1999 Bifurcations of one-dimensional stochastic differential equations. In Stochastic dynamics (ed. H. Crauel \& V. M. Gundlach), pp. 27-47. Springer.

Da Prato, G. \& Debussche, A. 1996 Construction of stochastic inertial manifolds using backward integration. Stoch. Stoch. Rep. 59, 305-324.

Debussche, A. 1998 Hausdorff dimension of a random invariant set. J. Math. Pures Appl. 77, 967-988.

Flandoli, F. \& Schmalfuss, B. 1996 Random attractors for the 3D stochastic Navier-Stokes equation with multiplicative white noise. Stoch. Stoch. Rep. 59, 21-45.

Foias, C., Sell, G. R. \& Teman, R. 1988 Inertial manifolds for nonlinear evolution equations. J. Diff. Eqns 73, 309-353.

Hale, J. K. 1988 Asymptotic behavior of dissipative dynamical systems. Mathematical Surveys and Monographs, vol. 25. Providence, RI: American Mathematical Society.

Henry, D. 1984 Geometric theory of semilinear parabolic equations. Springer Lecture Notes in Mathematics, vol. 840. Springer.

Kotelenez, P. 1992 Comparison methods for a class of function valued stochastic partial differential equations. Prob. Theory Relat. Fields 93, 1-19.

Ladyzhenskaya, O. A. 1991 Attractors for semigroups and evolution equations. Cambridge University Press.

Marion, M. 1987 Attractors for reaction-diffusion equations; existence and estimate of their dimension. Applicable Analysis 25, 101-147.

Pardoux, E. 1975 Équations aux dérivées partielles stochastiques non linéaires monotones. Thesis, University of Paris XI.

Renardy, M. R. \& Rogers, R. C. 1992 An introduction to partial differential equations. Springer. 
Robinson, J. C. 2001 Infinite-dimensional dynamical systems. Cambridge University Press.

Schmalfuß, B. 1992 Backward cocycles and attractors of stochastic differential equations. In Int. Seminar on Applied Mathematics - Nonlinear Dynamics: Attractor Approximation and Global Behaviour (ed. V. Reitmann, T. Riedrich \& N. Koksch), pp. 185-192. Dresden: Technische Universität.

Schmalfuß, B. 1996 A random fixed point theory based on Lyapunov exponents. Random Comput. Dynam. 4, 257-268.

Smith, H. L. 1995 Monotone dynamical systems. AMS Math. Surveys and Monographs, vol. 41. Providence, RI: AMS.

Temam, R. 1988 Infinite dimensional dynamical systems in mechanics and physics. Springer. 